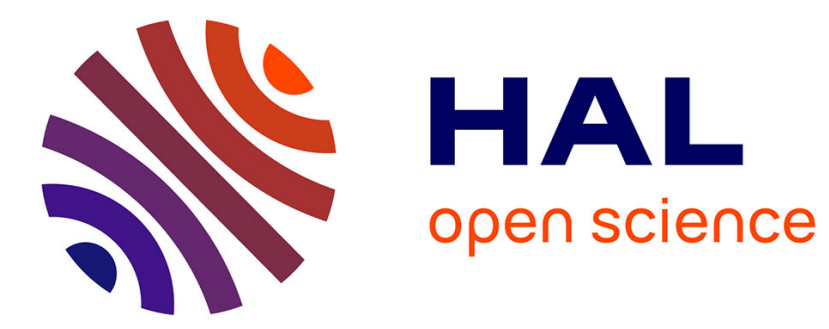

\title{
Familles à l'épreuve
}

Jean-Sébastien Eideliman

\section{To cite this version:}

Jean-Sébastien Eideliman. Familles à l'épreuve. Ethnologie française, 2009, 39 (3), 10.3917/ethn.093.0435 . hal-01241829

HAL Id: hal-01241829

https://hal.univ-lille.fr/hal-01241829

Submitted on 21 Dec 2015

HAL is a multi-disciplinary open access archive for the deposit and dissemination of scientific research documents, whether they are published or not. The documents may come from teaching and research institutions in France or abroad, or from public or private research centers.
L'archive ouverte pluridisciplinaire HAL, est destinée au dépôt et à la diffusion de documents scientifiques de niveau recherche, publiés ou non, émanant des établissements d'enseignement et de recherche français ou étrangers, des laboratoires publics ou privés.

$$
\text { Copyright }
$$




\section{Familles à l'épreuve}

(à paraître dans le numéro d'Ethnologie française 2009-3, « Handicaps. Entre discrimination et intégration »)

EIDELIMAN Jean-Sébastien - Centre Maurice Halbwachs, équipe «Enquêtes, terrains, théories » (ENS, EHESS, CNRS, Université de Caen)

\section{RESUME}

Dans une famille, la présence d'un adolescent présentant des difficultés intellectuelles importantes engendre des engagements au quotidien. L'enquête de l'ethnologue crée elle aussi des perturbations dans la famille, et cette situation peut être l'occasion d'aborder la question de la parenté sous un angle nouveau. Selon la manière dont l'enquête est accueillie, considérée, voire instrumentalisée, s'esquissent des formes de réaction à l'enquête. Elles correspondent aussi à des formes d'organisation familiale. La présente étude repose sur l'analyse de 42 monographies de famille. Elle permet de distinguer quatre formes d'acceptation de l'enquête. Celle-ci peut se réduire au point de vue d'un porte-parole (protection), elle peut donner l'occasion de tenter de mobiliser l'entourage (confrontation); elle peut s'orienter vers l'observation d'une forme de solidarité familiale (sélection) ou être accueillie avec docilité (compliance).

Mots-clés : Handicap mental. Enfance. Parenté. Ethnographie

Du fait des évolutions démographiques et comportementales, la famille est de plus en plus considérée comme un ensemble de relations en constante évolution [Singly, 1993] ${ }^{\mathrm{i}}$. Chaque événement familial peut entraîner une perturbation qui nécessite une recomposition, une réorganisation des échanges et des services familiaux [Déchaux et Herpin, 2004]. La mise en place de nouveaux arrangements va redéfinir les relations familiales comme l'illustrent une naissance [Orain, 2007], un mariage [Berger et Kellner, 1988], une séparation [Martial, 2003], un décès [Caradec, 2001]. Un enfant qui ne suit pas les normes de développement intellectuel éprouve profondément le mode de vie et la cohésion de la famille. Dans les discours des professionnels du handicap comme dans la presse, spécialisée ou non, le lien entre la présence d'un enfant handicapé et la probabilité d'un divorce ou d'une séparation des parents est fréquemment postulé. La perturbation supposée qu'entraîne un événement inattendu peut donner l'occasion à l'ethnologue d'observer la famille sous un nouvel angle. Il dépasse ainsi l'évidence à travers laquelle la famille s'offre au regard lorsque son fonctionnement effectif coïncide, au moins en apparence, avec son 
fonctionnement légitime, idéal [Bourdieu, 1993]. À l'épreuve du handicap mental, la famille offre ainsi une nouvelle prise à la réflexion sur le fait familial.

L'enquête ethnographique elle-même est parfois analysée en des termes semblables, c'est-àdire comme une perturbation révélatrice. Selon Olivier Schwartz [1993], l'ethnographe doit se défaire du fantasme d'observer ce qui se passerait en son absence et utiliser au contraire la perturbation qu'il provoque et les réactions qu'elle entraîne. Une condition s'impose cependant à lui : réduire cette perturbation pour la maintenir dans les limites du raisonnable, en jouant notamment sur le temps long de l'enquête. Ce sont donc deux perturbations que l'on observe lorsque l'on enquête sur des familles aux prises avec le handicap mental d'un adolescent. Il n'est pas toujours facile de les dissocier.

L'enquête sur laquelle je m'appuie ici ${ }^{\mathrm{ii}}$ s'est déroulée entre 2002 et 2005 et a porté sur 42 familles habitant l'Île-de-France. Elles ont été contactées via deux institutions : une école privée, accueillant des enfants handicapés mentaux âgés de 6 à 20 ans, et une association de parents et amis d'enfants handicapés mentaux. Chacune de ces familles compte donc un enfant (le plus souvent un adolescent), inséré dans le champ du handicap mental, bien que tous n'aient pas été administrativement reconnus comme handicapés mentaux. Sur ces 42 cas, j'ai élaboré des monographies de famille en multipliant les entretiens et les observations autour d'un même enfant ou adolescent. Cette méthode d'enquête a le mérite de multiplier des points de vue que l'on peut ensuite croiser et rapporter aux situations des uns et des autres. Mais elle nécessite de se « frayer un chemin » dans la famille, c'est-à-dire de convaincre les uns et les autres de participer à l'enquête. Concrètement, dans chaque cas, j'ai commencé par rencontrer une personne contactée par courrier, via une institution (soit une école pour enfants handicapés mentaux, soit une association de parents et amis de personnes handicapées mentales). Au terme de ce premier entretien, et en m'appuyant sur l'arbre de famille que je propose systématiquement d'établir, je demande à mon interlocuteur si d'autres personnes de l'entourage accepteraient de me rencontrer. Je réalise alors autour de chaque adolescent autant d'entretiens et d'observations qu'il est possible.

Cet article vise à analyser la double perturbation qu'engendrent dans une famille la présence d'un adolescent handicapé mental et, sur un temps plus court, celle d'un ethnologue. Je voudrais montrer que la réflexivité que l'ethnologue se doit de mener par rapport à son enquête est, ici plus qu'ailleurs, étroitement liée aux résultats mêmes de l'enquête. En d'autres termes, le retour réflexif sur les conditions de réalisation de l'enquête m'en ont autant appris sur les organisations familiales que j'analysais que le contenu des entretiens et des observations que j'ai pu mener. J'étudierai successivement ces deux perturbations, afin de montrer combien ces deux dimensions peuvent être intimement liées dans l'analyse. 


\section{À L’ÉPREUVE DU HANDICAP MENTAL}

De nombreux travaux ont énuméré les éléments qui font peser sur l'entourage des personnes âgées dépendantes [Clément et Lavoie, 2005], malades mentales [Bungener, 1995] ou encore handicapées [Triomphe, 2003] un poids que beaucoup qualifient de «fardeau (burden en anglais) des aidants » [Biegel, 2001]. Dans le cas des enfants handicapés mentaux, les charges qui peuvent peser sur les membres impliqués de l'entourage, à des degrés très différents selon la nature des difficultés et les configurations sociales et familiales, sont les suivantes : coûts en argent (réduction du temps de travail d'un des parents, appareillage éventuel, transports, garde), en temps (surveillance continuelle, aide à la vie quotidienne, démarches institutionnelles multiples, accompagnement de l'enfant à divers rendez-vous), en investissement psychique (décisions à prendre pour l'enfant, angoisses pour son avenir, regard pesant des autres, culpabilité), en répercussions sur les relations familiales et les autres membres de la famille (dégradation de l'ambiance familiale, difficultés scolaires ou psychiques des frères et sœurs, tensions entre les parents). Ces divers éléments ne sont bien sûr pas systématiquement présents et peuvent en outre être partiellement compensés par des retombées positives de l'investissement auprès de l'enfant. Mais ils permettent d'imaginer que des difficultés intellectuelles importantes chez un enfant peuvent engendrer toutes sortes de bouleversements dans les fonctionnements familiaux.

Pour saisir ces bouleversements, on peut s'appuyer sur une distinction qui oppose les liens formels de parenté aux relations de parenté [Eideliman, 2003]. Les premiers découlent directement de la hiérarchisation de la famille en degrés de parenté, tandis que les relations de parenté renvoient à la réalité des comportements quotidiens entre membres d'une même famille. Selon le lien formel de parenté que l'on entretient avec une personne de sa famille (père, mère, enfant, grand-parent, belle-mère, demi-sœur etc.), un ensemble de comportements sont attendus, voire juridiquement exigés. Ces liens formels sont partiellement hiérarchisés et les obligations sont d'autant plus fortes et nombreuses que l'on se situe au haut de la hiérarchie (filiation, alliance, germanité). Ce sont les liens que l'on représente lorsque l'on construit des arbres de famille et c'est d'eux que découle la terminologie de parenté. Mais entre les prescriptions du système d'obligations matérielles et morales qu'organise la parenté et la réalité des comportements, que j'appelle des relations de parenté, il y a un écart sur lequel joue tout un ensemble de facteurs tels que la communication, la fréquence des contacts et leur nature, les échanges de biens et services, la géographie familiale ou encore les relations affectives.

Cet écart est bien connu des sociologues et surtout des anthropologues. Raymond Firth et Judith Djamour [1956] pointent ainsi dès les années 1950 la différence entre proximité attendue et proximité affichée entre membres de la parenté. Cette distinction rappelle aussi celle que Claude Lévi-Strauss [1958] expose dans les mêmes années entre système des appellations (ce que l'on doit 
dire pour parler des membres de sa parenté) et système des attitudes (ce que l'on doit faire vis-à-vis des membres de sa parenté). L'objectif de l'opposition entre liens formels et relations de parenté vise davantage à souligner la différence entre ce que l'on doit dire ou faire et ce que l'on dit ou fait, plutôt qu'entre ce que l'on doit dire et ce que l'on doit faire. Cette différence est donc plus proche de l'optique de Raymond Firth et Judith Djamour [ibid.], ou encore de celle de Pierre Bourdieu [2002 : 174] lorsqu'il critique le «juridisme », que de celle de Claude Lévi-Strauss [ibid.]. Enfin, la volonté d'articuler liens formels et relations de parenté, de comprendre la manière dont les secondes s'éloignent ou se rapprochent des premiers, justifie cette distinction bien plus que la séparation de deux ordres de phénomènes.

Ce travail interne à la parenté, ces bouleversements, ces «arrangements pratiques » [Eideliman et Gojard, 2008] accompagnent les événements familiaux (dont font partie l'arrivée et l'évolution d'un enfant ayant des difficultés intellectuelles). Ils composent l'objet central de mon analyse. Comment les relations de parenté s'adaptent-elles aux difficultés de l'enfant ? En fonction de quoi évoluent-elles ? Comment se détermine et évolue la participation de chacun ? Bref, comment les membres d'une même famille «s'arrangent»-ils, comment composent-ils avec les problèmes particuliers que posent les difficultés intellectuelles d'un enfant ou adolescent, et comment retissent-ils les relations de parenté qu'ils entretiennent entre eux au quotidien ? Plutôt que d'exposer en détail ces mécanismes [Eideliman, 2008], je voudrais montrer que l'examen réflexif du déroulement de l'enquête dans une famille permet d'entrer assez profondément dans ces fonctionnements, même si l'utilisation de l'ensemble du matériau recueilli permet bien sûr d'aller plus loin dans l'analyse.

\section{À L'ÉPREUVE DE L'ENQUÊTE}

Dans le déroulement de mes monographies de famille, le fait marquant est que, sur les 42 cas analysés, 39 fois j'ai eu affaire en tout premier à la mère de l'enfant (par téléphone puis pour un entretien). La norme de l'implication particulière de la mère vis-à-vis des enfants apparaît ici de manière éclatante. On peut dire qu'en matière d'éducation des enfants handicapés mentaux, ce sont les mères qui sont en «première ligne ». Cela ne signifie pas qu'elles sont forcément les plus investies, mais que c'est sur elles que pèse le plus lourdement l'exigence du care [Finch et Groves, 1983]. Dans certains cas, elles peuvent partager cette «première ligne » avec d'autres, le plus souvent le père de l'enfant, parfois leur propre mère, parfois leur nouveau conjoint, bien plus rarement une sœur ou une amie. Dans d'autres cas, elles sont seules à occuper cette position avancée. Au-delà des parents de l'enfant, l'orientation que la première personne rencontrée donne à l'enquête permet de bâtir une typologie révélatrice des réactions à l'enquête. 


\section{$\underline{\text { Adopter ou exclure }}$}

Dans de nombreux cas, la première personne rencontrée (la mère en général) dirige le chercheur vers ceux qui font partie de la maisonnée [Weber, 2002 et 2005] constituée autour de l'enfant, c'est-à-dire le groupe de production domestique. Il s'organise au quotidien pour assurer la survie de ses membres dans les meilleures conditions possibles. L'enquêteur est donc dirigé vers les «adoptés » et tenu à l'écart des «exclus », c'est-à-dire de ceux qui ne participent pas à la maisonnée, quand bien même leur lien formel de parenté avec l'enfant supposerait une plus grande proximité. L'exemple du cas de Camille Briolle ${ }^{\mathrm{iii}}$, une jeune fille présentant divers troubles associés (malvoyance, retard psychomoteur, difficultés d'élocution, difficultés intellectuelles), illustre bien cette première attitude. Elle vit avec ses trois frères et sœurs et ses parents en banlieue parisienne (sa mère, Michèle Briolle, a été kinésithérapeute et son père, Hervé Briolle, est ingénieur).

Dans le cas des Briolle, la façon dont se déroulent les entretiens est singulière. Chaque fois (en février 2004 et en mai 2005), Michèle Briolle utilise une réunion de famille pour me permettre de faire plusieurs entretiens d'affilée, chez eux, sans toujours avertir les différentes personnes de ma venue ni de mon travail. Elle semble estimer que ceux qui participent à ces réunions de famille connaissent bien Camille et sont donc tout désignés pour participer à mon enquête. Elle instaure en ma faveur une forme de «travail ethnographique à la chaîne », m'installant dans un bureau où vont défiler les différentes personnes présentes ce jour-là ${ }^{\mathrm{iv}}$. 


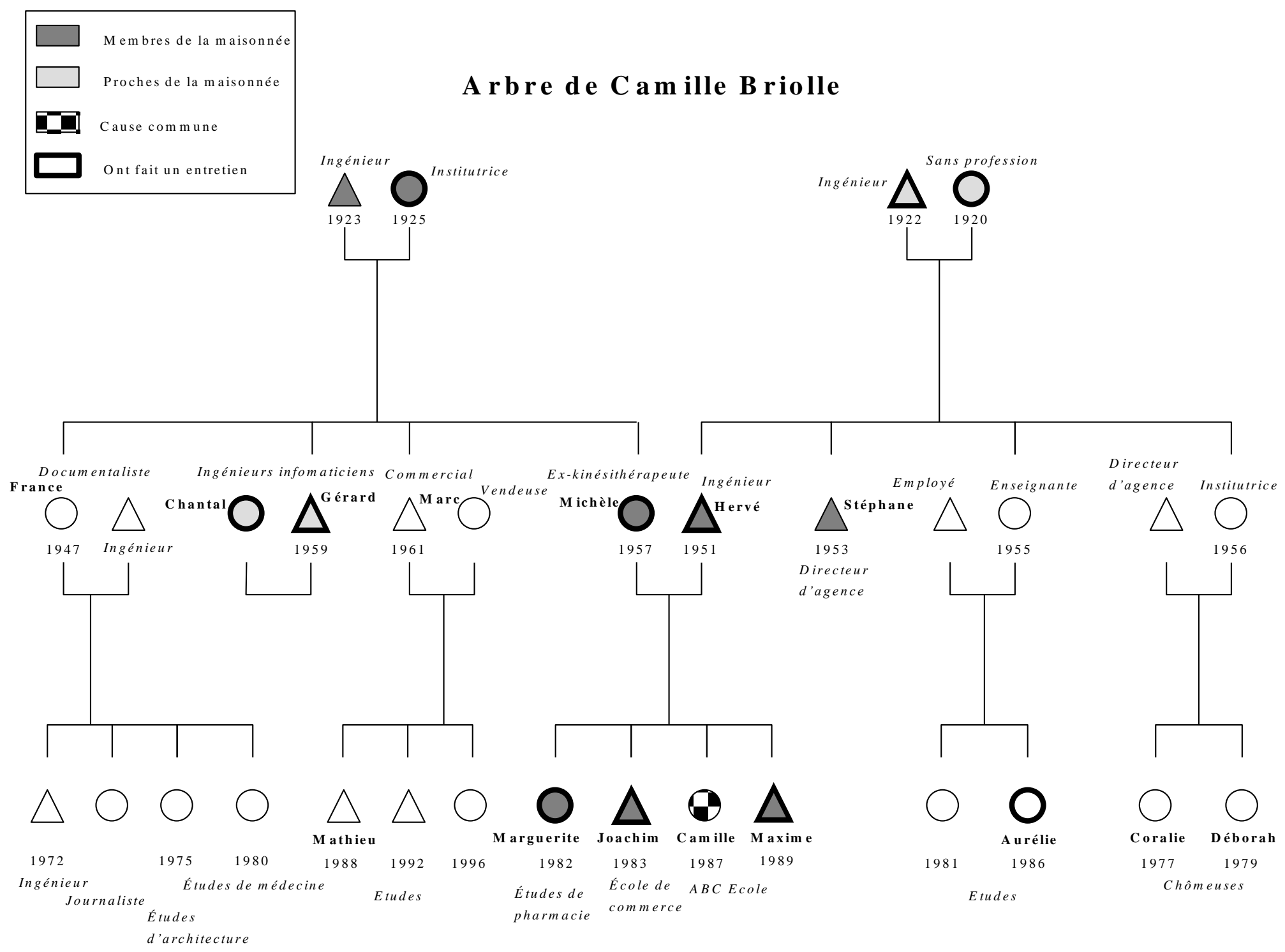


L'un des frères de Michèle, Marc, n'était présent à aucune de ces deux réunions. J'aurais aimé connaître son point de vue : je tente d'insister auprès de la mère de Camille. Sans succès. Elle invoque différentes raisons (il habite loin, il est malade), pour finalement signaler le différend qui les oppose et qui porte en partie sur Camille. Marc estime que les parents de Camille en font trop pour elle, sollicitent excessivement ses grands-parents maternels (au détriment des enfants de Marc), alors qu'ils feraient mieux de la confier à un institut. Je ne pourrai donc pas proposer un entretien à Marc, exclu des réunions de famille et, du même coup, de mon enquête. Pour voir Marc, il faudrait en effet desserrer un double blocage. Celui de Michèle qui n'estime pas que son point de vue sur Camille soit légitime. Mais aussi peut-être celui de Marc, dont on peut penser qu'il refuserait de répondre à un enquêteur que sa sœur lui adresserait. Ne lui demanderait-il pas de parler de Camille, sur laquelle il porte un jugement qui n'est pas forcément facile à assumer devant un ethnologue, étudiant les situations de handicap? Dans le cas de l'enquête autour d'un autre adolescent, Axel Costa, j'ai d'ailleurs essuyé un refus de même nature : la mère d'Axel avait accepté sans grand enthousiasme de me donner les coordonnées d'une de ses sœurs, avec laquelle elle ne s'entendait pas très bien. Cette dernière a d'abord accepté de réaliser l'entretien avec moi, avant de l'annuler par texto. Contactée par téléphone, elle a ensuite refusé un nouveau rendez-vous.

Bien souvent, l'enquête chemine ainsi au sein de la maisonnée, c'est-à-dire entre les personnes les plus impliquées vis-à-vis de l'enfant. Celles qui sont moins investies, voire exclues, sont jugées illégitimes par les membres de la maisonnée. Ces personnes n'ont pas à s'exprimer au sujet de l'enfant. De plus, elles sont généralement conduites à refuser toute enquête, potentiellement accusatrice, et qu'elles perçoivent de plus comme relevant d'un groupe dont elles ne font pas partie. Si l'on pousse plus loin l'analyse - c'est le cas des Briolle - on observe qu'autour des difficultés de Camille se réactualisent entre Michèle et Marc des conflits aux multiples enjeux, fondés sur divers éléments de leur histoire familiale. Autour de Camille, les Briolle poussent au maximum la logique de maisonnée et attendent donc de leur entourage un dévouement qui dépasse la logique de la répartition égalitaire des ressources familiales. Marc refuse cette logique. Il se plaint de ce que les parents de Camille attirent à eux des ressources dont il se trouve alors privé (à commencer par le temps des grands-parents, c'est-à-dire les parents de Marc et Michèle). Sa manière de concevoir les difficultés et les besoins de Camille est influencée par cette position : il trouve que Camille ne peut plus vraiment progresser et serait mieux à sa place dans un institut qui la prendrait entièrement en charge. Pour peu qu'on y prête attention, mon cheminement dans cette famille révèle ces logiques et ces conflits. 


\section{Protéger ou confronter}

Cependant, les réactions d'une famille à l'enquête peuvent répondre à une autre logique. Je n'ai réussi à mener autour de Gaultier Villeneuve, qui est atteint de trisomie 21, que trois entretiens... avec une seule et même personne : sa mère, Marianne Villeneuve (sans profession, ancienne directrice commerciale). Son mari est ingénieur informaticien, et leur niveau de vie est très élevé. Ce n'est pourtant pas faute d'avoir essayé : en octobre 2004, à la fin de notre premier entretien, j'explique comme d'habitude à Marianne Villeneuve que j'aimerais parler de Gaultier avec d'autres membres de son entourage. Comme une bonne partie de sa famille habite à Paris ou en proche banlieue, les candidats potentiels sont nombreux. Lors de notre deuxième entretien, toujours en octobre 2004, Marianne m'annonce qu'il sera difficile de voir quelqu'un d'autre, car tous sont très occupés. Dans les mois qui suivent, je relance régulièrement (à quatre reprises) Marianne Villeneuve. Chaque fois elle me répond qu'elle n'a pas encore eu le temps d'en parler autour d'elle ou que ce n'est pas le bon moment. Finalement, en mai 2005, elle essaie de mettre un terme à ma requête d'une façon originale, en me proposant de me restituer elle-même «objectivement» le point de vue de chacun. Marianne Villeneuve s'érige donc en rempart entre moi et ses proches. En revanche, elle m'incite vivement à contacter des personnes plus éloignées de Gaultier, hors du cadre familial, comme son enseignante spécialisée ou une mère d'enfant handicapé militante. À l'inverse de Michèle Briolle, Marianne Villeneuve m'envoie donc vers des personnes moins investies autour de Gaultier, tout en me détournant des plus proches, dont elle se propose même, pour faire face à mon insistance, de prendre la place.

Le cas d'Emma Cauwelaert peut sembler identique au premier abord. Emma est également atteinte de trisomie 21. Ses parents (le père est assureur et la mère, infirmière) habitent un petit appartement dans un quartier chic de Paris. Là encore, je n'ai pu voir que Béatrice, sa mère. Chaque fois que je la rappelais pour savoir si d'autres personnes de l'entourage d'Emma seraient d'accord pour me voir, Béatrice me répondait qu'elle leur en avait un peu parlé mais qu'ils ne disaient ni oui, ni non, et qu'elle ne les sentait donc pas prêts. Cependant, en prêtant plus d'attention à la manière dont Béatrice présente les choses, on se rend compte qu'on est en fait bien loin d'une attitude de protection. Béatrice se montre d'abord très intéressée par mon enquête, aussi bien pendant notre entretien de septembre 2004, qu'au cours de mes relances téléphoniques. Elle m'assure en avoir beaucoup parlé autour d'elle, mais qu'on lui répond toujours « oui » sans conviction, sans lui dire vraiment qu'elle peut me transmettre des coordonnées. En mai 2005, alors que je la rappelle pour la énième fois, elle laisse éclater son amertume : 


\section{Journal de terrain, le 18/05/05: relances téléphoniques}

Mme Cauwelaert me confirme que les membres de sa famille ne sont pas disponibles pour mon enquête. 'Ils sont tous en paroles.', lâche-t-elle. C'était ce qu'elle craignait. Ils ont peut-être peur de ne pas savoir quoi dire. Mais selon elle, 'si on n'en parle jamais, rien ne se fera jamais.' Elle-même était enthousiaste par rapport à mon enquête, mais elle n'a pas réussi à les convaincre. Ils donnent des prétextes. Elle tend des perches, mais personne ne les attrape.

Malgré les apparences, Béatrice est dans une attitude inverse de la protection : elle cherche à confronter, sans succès, son entourage au handicap d'Emma. Mon enquête devient pour elle le moyen d'amener ses proches à réfléchir à la situation, de les pousser à s'impliquer, au moins par la pensée, dans la maisonnée qui entoure Emma, alors que celle-ci se réduit, selon Béatrice, essentiellement à elle-même et à son mari. Ce qu'elle déplore. La formule qu'elle utilise, « ils sont tous en paroles », exprime bien son sentiment d'être la seule à réellement agir pour Emma.

Dans le cas d'Emma, la volonté d'une mère de confronter l'entourage au handicap de sa fille, grâce à mon enquête, a été un échec: elle n'a réussi à décider aucune autre personne à participer; d'où l'amertume de cette mère. La démarche de confrontation aboutit parfois à un tout autre résultat, comme dans le cas de Tristan Mareste (également atteint de trisomie 21). Sa mère, Fabienne (elle est responsable technique dans une agence de publicité ; son mari est directeur administratif et financier), s'était aussi engagée dans une démarche active de confrontation et était parvenue à décider seize membres de l'entourage de Tristan à participer à mon enquête, dont une majorité de personnes peu ou pas investies.

Dans le cas des Villeneuve, on a donc une configuration bien différente de celle des Briolle, qui transparaît là encore dans le cheminement de l'enquêteur dans la famille. S'appuyant sur de solides ressources économiques et sociales, Marianne Villeneuve prend en charge son fils à elle toute seule (en s'appuyant sur des professionnels). Elle protège les membres de l'entourage en leur laissant un rôle secondaire au quotidien, tout en les valorisant devant l'enquêteur. Du côté de Béatrice Cauwelaert en revanche, le fait qu'elle manque de soutien pour s'occuper de sa fille est ressenti durement, mais elle ne parvient pas à trouver le moyen d'impliquer davantage son entourage dans l'organisation familiale restreinte mise en place autour d'Emma.

\section{Une typologie des réactions à l'enquête}

En m'appuyant sur l'ensemble du matériau, il est possible de distinguer quatre formes de réaction à l'enquête. Elles s'apparient à des formes distinctes d'organisation familiale.

- Protection (exemple : Gaultier Villeneuve) : la mère (puisque c'est presque toujours le premier contact) bloque l'enquête ou la limite à un cercle très restreint. On peut faire l'hypothèse que ces situations sont majoritairement le fait de familles appartenant aux 
classes supérieures, qui semblent les plus à même et les plus désireuses de se protéger vis-à-vis d'une enquête perçue comme intrusive. Un cas typique est celui où la mère ne travaille pas, s'occupe intensément de l'enfant handicapé et considère qu'elle est la seule à posséder une parole légitime à son sujet. Elle se pose alors en médiateur entre l'enquêteur et la famille. Sur les 42 cas étudiés, 17 correspondent à cette situation et ont donné lieu en moyenne à 2,3 entretiens chacun ( 1,8 si on ne compte que les entretiens réalisés avec des personnes différentes).

- Confrontation : la mère, qui se sent trop seule pour s'occuper de l'enfant dit handicapé, se saisit de l'enquête pour impliquer largement les membres de l'entourage et les faire réfléchir à la situation, voire leur faire prendre position. Lorsque cette tactique emporte le succès (exemple : Tristan Mareste), on peut faire l'hypothèse qu'elle est le propre des familles où le premier contact est en position de force et dispose de suffisamment de ressources et de pouvoir (du fait par exemple de sa position professionnelle ou de son niveau d'étude) pour imposer l'enquête à un grand nombre de membres de son entourage. 3 cas correspondent à cette configuration et ont donné lieu en moyenne à 12 entretiens chacun (10,7 si on ne compte que les entretiens effectués avec des personnes différentes). Lorsque cette tactique se heurte à l'échec (exemple : Emma Cauwelaert), il s'agit plutôt de cas où le premier contact est en position de faiblesse dans la famille. 2 cas correspondent à cette configuration et ont donné lieu en moyenne à 5 entretiens chacun $(3,5$ si on s'en tient aux entretiens réalisés avec des personnes différentes).

- Sélection (exemple : Camille Briolle) : la mère de l'enfant dit handicapé ne se plie pas aux directives de l'enquêteur et opère elle-même une sélection au sein de son entourage. Elle l'oriente vers les personnes les plus investies auprès de l'enfant, c'est-à-dire vers ses partenaires au sein de la maisonnée formée autour de lui, qui acceptent très souvent de participer à l'enquête. En revanche, les personnes «exclues» de la maisonnée restent inaccessibles à l'enquêteur. Ces familles sont souvent fières de leur fonctionnement en maisonnée et peuvent appartenir à tous les milieux sociaux. Les différences de position et les rapports de pouvoir au sein de la famille peuvent exister, mais sont masqués par un travail de solidarisation du groupe familial. L'enquête devient pour eux l'occasion de mettre en avant cette solidarité. 10 cas correspondent à cette situation et ont donné lieu en moyenne à 5,9 entretiens (4,9 si on ne retient que les entretiens effectués avec des personnes différentes). 
- Compliance (exemple: Axel Costa) : la mère de l'enfant accueille favorablement l'enquête et se rend aux demandes de son auteur. Elle accepte de proposer l'enquête aux personnes indiquées par le chercheur, même celles qu'elle juge illégitimes pour parler de l'enfant. En revanche, les acceptations des membres de l'entourage ne sont pas forcément nombreuses, soit parce que cet entourage est peu important du fait d'un isolement relationnel, soit parce que les personnes peu impliquées déclinent la proposition. On retrouve ici les familles issues des milieux sociaux les moins favorisés, qui ont une moindre propension à résister à l'enquête et à l'enquêteur. 10 cas appartiennent à cette catégorie, qui ont donné lieu en moyenne à 4,4 entretiens chacun (2,9 si on ne compte que les entretiens effectués avec des personnes différentes).

J'ai construit ma réflexion à partir des réactions de personnes confrontées à l'éducation d'un adolescent ayant des problèmes consécutifs à un handicap mental. Réactions aux difficultés de l'enfant elles-mêmes, qui entraînent la mise en place d'arrangements pratiques au quotidien, mais aussi réactions à ma demande de parole sur ce sujet, qui déclenche un processus de réflexivité. Les uns l'accueillent avec empressement, d'autres avec méfiance. C'est alors que se perçoivent différentes organisations familiales : de la mère seule, qui assume la quasi-totalité de l'entretien de l'adolescent dit handicapé, jusqu'au groupe de production domestique, qui prend en charge collectivement un adolescent devenant une «cause commune » [Gollac, 2003], en passant par des mères qui se sentent trop seules face au poids quotidien que représente le handicap. 


\section{BIBLIOGRAPHIE}

Béliard Aude et Eideliman Jean-Sébastien, 2008, «Au-delà de la déontologie. Anonymat et confidentialité dans le travail ethnographique », in Bensa Alban et Fassin Didier (dir.), Politiques de l'enquête, Paris, La Découverte, à paraître

Berger Peter et Kellner Hansfried, 1988, «Le mariage et la construction de la réalité », Dialogue, $102: 6-21$

Biegel David, 2001, «Le fardeau des aidants », in Fondation Médéric Alzheimer, Les aidants familiaux et professionnels : de la charge à l'aide, Paris, Serdi Edition : 9-11

Bourdieu Pierre, 1993, « À propos de la famille comme catégorie réalisée », Actes de la recherche en sciences sociales, $100: 32-36$

Bourdieu Pierre, 2002, Le Bal des célibataires, Paris, Seuil

Bungener Martine, 1995, Trajectoires brisées, familles captives. La maladie mentale à domicile, Paris, Inserm

Caradec Vincent, 2001, « Le veuvage, une séparation inachevée », Terrain, $36: 69-84$

Clément Serge et Lavoie Jean-Pierre (dir.), 2005, Prendre soin d'un proche âgé. Les enseignements de la France et du Québec, Ramonville Sainte-Agne, Érès

Déchaux Jean-Hugues et Herpin Nicolas, 2004, «Entraide familiale, indépendance économique et sociabilité », Économie et statistique, $373: 3-32$

Eideliman Jean-Sébastien, 2003, «Exclusions, adoptions et relations de parenté », in Weber Florence, Gojard Séverine et Gramain Agnès (dir.), Charges de famille. Parenté et dépendance dans la France contemporaine, Paris, La Découverte : 312-361

Eideliman Jean-Sébastien, 2008, «'Spécialistes par obligation'. Des parents face au handicap mental. Théories diagnostiques et arrangements pratiques », thèse de sociologie sous la direction de Florence Weber, Paris, ENS/EHESS

Eideliman Jean-Sébastien et Gojard Séverine, 2008, « La vie quotidienne à domicile des personnes handicapées ou dépendantes : du 'besoin d'aide' aux arrangements pratiques' », Retraite et société, $53: 89-111$

Finch Janet and Groves Dulcie (dir.), 1983, A Labour of Love : Women, Work and Caring, Londres, Routledge Kegan Paul

Firth Raymond et Djamour Judith, 1956, « Kinship in South Borough », in Firth Raymond (dir.), Two Studies of Kinship in London, London, Athlone Press : 33-63

Gollac Sibylle, 2003, «Maisonnée et cause commune : une prise en charge familiale », in Weber Florence, Gojard Séverine et Gramain Agnès (dir.), Charges de famille. Parenté et dépendance dans la France contemporaine, Paris, La Découverte : 274-311 
Lévi-Strauss Claude, 1958, Anthropologie structurale, Paris, Plon

Martial Agnès, 2003, S'apparenter. Ethnologie des liens de familles recomposées, Paris, Éditions de la Maison des Sciences de l'Homme

Orain Renaud, 2007, «La naissance du Foyer ? Conditions matérielles des entrées dans les carrières parentales et de l'accueil du premier enfant en France à la fin du XXe siècle », thèse de sociologie sous la direction de Florence Weber, Université Paris VIII Vincennes-Saint-Denis

Schwartz Olivier, 1993, «L'empirisme irréductible. La fin de l'empirisme ? », postface à Anderson Nils, Le Hobo. Sociologie du sans-abri, Paris, Nathan : 265-308

Singly François (de), 1993, Sociologie de la famille contemporaine, Paris, Nathan

Triomphe Annie, 2003, «Les conditions de vie des personnes handicapées : premiers résultats à partir de l'enquête HID », Revue française des affaires sociales, 1-2 : 167-180

Weber Florence, 2002, « Pour penser la parenté contemporaine », in Debordeaux Danielle, Strobel Pierre (dir.), Les Solidarités familiales en questions. Entraide et transmission, Paris, LGDJ : 73-106 Weber Florence, 2005, Le Sang, le nom, le quotidien. Une sociologie de la parenté pratique, La Courneuve, Aux lieux d'être

\section{ABSTRACT}

Families put to test

Having a teenager with learning disabilities in a family entails a reorganization of everyday life. From the ethnologist's viewpoint, whose investigation creates some disturbances in the family, this situation may be a chance to tackle kinship from another perspective. The ways the research was received, regarded or even used by the participants can be analysed as different ways of reacting, which also correspond to patterns of organization. Drawing on 42 family case studies, the research presents four different modes of accepting it: it can be brought down to the viewpoint of a spokesperson (protection); it can be used as an opportunity to attempt to mobilize the family circle (confrontation); it can be geared towards observing a form of family solidarity (selection); or it can be received with docility (compliance).

Keywords : Mental Disability. Childhood. Kinship Ethnography

\section{ZUSAMMENFASSUNG}

Familien auf Probe 
Die Gegenwart in einer Familie eines Halbwüchsigen mit bedeutenden intellektuellen Schwierigkeiten bringt mit sich Neugestaltungen der täglichen Familienorganisation. Aus der Sicht des Ethnologen, dessen Studie auch Störungen in der Familie mit sich zieht, gibt diese Sachlage die Gelegenheit, die Verwandschaft aus einer neuen Sicht zu studieren. Die Reaktionen der Familien auf die Untersuchung stehen mit Familienorganisationsformen zusammen in Verbindung. Die Studie, die hier präsentiert wird, stützt sich auf 42 Monografien, und ermöglicht es, vier verschiedenen Akzeptierungsweisen zu unterscheiden. Die Untersuchung kann sich auf die Sichtweise eines Sprechers beschränken (Beschützung) ; sie kann die Gelengenheit bieten, die Angehörigen mobilisieren zu versuchen (Gegenüberstellung) ; sie kann auf die Beobachtung eines Familiengemeinschaftsgeists gerichtet werden (Auslese); sie kann schließlich mit Folgsamkeit empfangen werden (Komplianz).

Stichwörter : Behinderung geistig Kindheit Verwandtschaft Ethnographie

\footnotetext{
i Je remercie vivement l'ensemble de l'équipe MEDIPS (Modélisation de l'économie domestique et incidence des politiques sociales), dont les travaux ont largement inspiré cet article, et en particulier Aude Béliard pour ses relectures et suggestions. Je tiens également à exprimer tous mes remerciements à Marjolaine Roger et Marin Sirakov pour leur aide précieuse.

ii Cette enquête a été menée dans le cadre d'une thèse de sociologie [Eideliman, 2008] dirigée par Florence Weber, soutenue en octobre 2008.

iii Tous les noms de lieux et de personnes cités ci-dessous ont été anonymisés. De plus, afin de respecter la confidentialité des informations données par une personne par rapport à ses proches, qui ont parfois eux aussi participé à l'enquête, certaines caractéristiques des cas présentés ont été modifiées à partir de celles d'un autre cas. J'ai justifié ailleurs [Béliard et Eideliman, 2008] de telles pratiques d'anonymisation, qui ne sont nécessaires que dans des cas particuliers où des problèmes de confidentialité, plus que d'anonymat, sont en jeu.

${ }^{\text {iv }}$ Voir sur l'arbre les différentes personnes avec qui j'ai pu avoir un entretien.
} 